\title{
Intra Subject Variability
}

National Cancer Institute

\section{Source}

National Cancer Institute. Intra Subject Variability. NCI Thesaurus. Code C67498.

The range of possible values for any measurable characteristic, physical or mental, of a human being, analyzed, examined, investigated, experimented upon, or treated in the course of particular study. 